\title{
2D phase unwrapping using Markov random field based phase locked loops
}

\author{
N. Deniz Cagatay and Mihai Datcu \\ German Aerospace Center (DLR) \\ Remote Sensing Technology Institute \\ 82234 Oberpfaffenhofen, Germany
}

\begin{abstract}
In this work, a Markov random field based phase locked loop is proposed for phase unwrapping. The neighboring pixels are used to update the phase estimate of the centering pixel. The performance of the proposed method is evaluated for both synthetic and real interferometric phase. For terrains with relatively low slopes, the phase unwrapping is done successfully. However, in case of high fringe frequency, the method fails to unwrap the whole phase gradient. Nevertheless, the noise suppression capability of phase locked loop is remarkable.
\end{abstract}

Keywords: Phase unwrapping, Markov random fields, phase locked loops

\section{INTRODUCTION}

Synthetic Aperture Radar (SAR) interferometry makes use of the phase difference between two complex SAR images acquired from slightly different positions in order to derive surface topography. Given two registered complex SAR images of the same scene, $z_{1}$ and $z_{2}$, the terrain height is related to the absolute phase of the interferogram, i.e., $\varphi$.

$$
\begin{aligned}
z_{1} & =\alpha_{1} \cdot e^{j \varphi_{1}} \\
z_{2} & =\alpha_{2} \cdot e^{j \varphi_{2}} \\
I=z_{1} \cdot z_{2}^{*} & =\alpha_{1} \cdot \alpha_{2} \cdot e^{j\left(\varphi_{1}-\varphi_{2}\right)} \\
\varphi=\arg (I) & =\varphi_{1}-\varphi_{2}
\end{aligned}
$$

However, the $\arg ($.$) operator which is used to extract the interferometric phase corresponds to modulo 2 \pi$ operation. Hence, the observed interferometric phase is wrapped over the principle interval $[\pi,-\pi)$. Besides, the observed phase is corrupted by noise. Phase unwrapping problem is the reconstruction of the absolute phase signal from its observed noisy wrapped samples by removing $2 \pi$ ambiguities.

On the other hand, a phase locked loop (PLL) is a control system which generates an output signal whose phase is related to the phase of the input signal. Here, the idea is to make use of this circuitry which has been widely used in telecommunications as a phase unwrapping algorithm. In this work, Markov random field approach is adopted to the already existing PLL phase unwrapping algorithms. ${ }^{1,2}$

The paper is organized as follows. In Sec. 2, operation principle of PLL is explained in terms of phase unwrapping. Section 3 introduces Markov random field based PLLs proposed for phase unwrapping. In Sec. 4, the experimental results are presented for both synthetic and real interferometric data. Finally, the results are concluded in Sec. 5.

Further author information: (Send correspondence to N.D.C.)

N.D.C.: E-mail: nazli.kahyaoglu@dlr.de,

M.D.: E-mail: mihai.datcu@dlr.de 


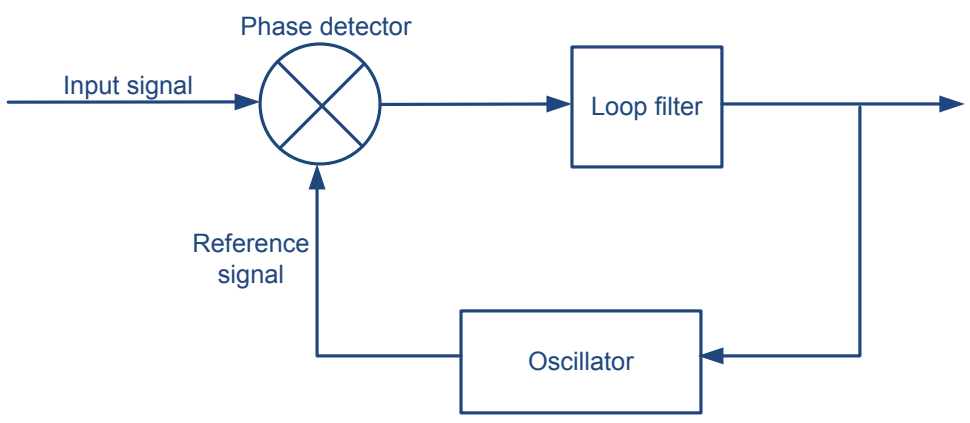

Figure 1. Phase locked loops

\section{PHASE UNWRAPPING USING PHASE LOCKED LOOPS}

PLL is a feedback loop which mainly consists of a phase detector, a loop filter and an oscillator (See Fig. 1). The phase detector compares the input phase with the reference phase synthesized by the oscillator, and generates an error measure which depends on the phase difference. The loop filter integrates these errors and the oscillator updates the reference phase accordingly.

As stated above, phase unwrapping is the reconstruction of the absolute phase signal from its observed noisy wrapped samples. However, the solution is not unique, and some smoothness or regularization assumptions (i.e., an a-priori model) are required. ${ }^{4}$ From the Bayesian point of view, the problem can be stated as the estimation of the original phase from the wrapped samples given a-priori knowledge of the original phase, i.e.,

$$
\begin{gathered}
\hat{\varphi}=\arg _{\varphi} \max \{\text { likelihood }\} \\
\varphi \in\{\text { a-priori model }\}
\end{gathered}
$$

Considering the PLL output as a maximum a-posteriori (MAP) estimate, it may be used for phase unwrapping. Indeed, PLL has already been proposed to unwrap the two dimensional interferometric phase. ${ }^{1,2}$ As opposed to common unwrapping methods dealing with the wrapped phase obtained by the $\arg ($.$) operator, PLL operates on$ the complex interferogram directly, and it continuously tracks and demodulates the phase of the input signal by integrating the estimation errors through the loop filter and the oscillator.

\section{MARKOV RANDOM FIELD BASED PLL FOR PHASE UNWRAPPING}

In the literature, the phase unwrapping techniques which make use of PLL ${ }^{1,2}$ integrate the phase errors in a row-by-row or column-by-column manner, which imposes causality. Then, in two dimensional (2D) case, the past (P) and future (F) may be represented as in Fig. 2(a) and Fig. 2(b). A non-causal approach may also be adopted by means of Markov random fields.

Markov random field (MRF) is a well-known appropriate statistical model for parameter estimation of images. ${ }^{3}$ In this model, Markovianity condition states that the conditional probability of a state $s$ in an $M \times N$ lattice having the value of $z_{s}$ depends only on the neighboring states, i.e.,

$$
P\left(z_{s} \mid z_{1}, z_{2}, \ldots, z_{s-1}, z_{s+1}, \ldots, z_{M \times N}\right)=P\left(z_{s} \mid z_{t} ; t \in N_{s}\right),
$$

where sites $t \in N_{s}$ represent the neighbors of site $s$ in an $M \times N$ lattice. For instance, Fig. 2(c) illustrates a fourth order neighborhood.

Based on this model, an MRF-based PLL is proposed to be used for 2D phase unwrapping, and, for a better noise suppression and tracking capability, a second order PLL as in Fig. 3 is implemented in this work.

In this approach, considering the second order neighboring pixels as the past based on the MRF model, the initial phase estimate of the center pixel is first compared with the observed phase values of 8 neighboring pixels. 


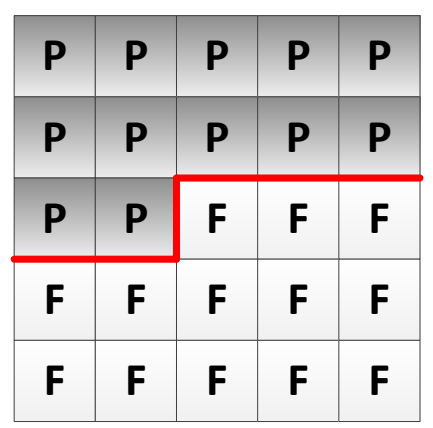

(a)

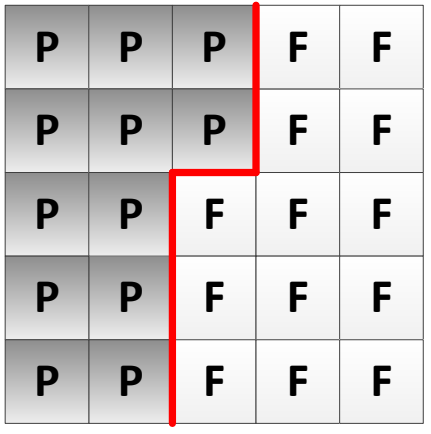

(b)

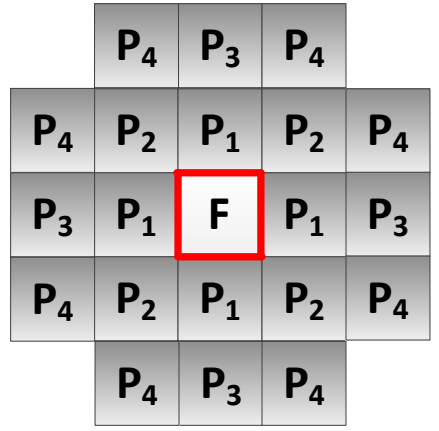

(c)

Figure 2. (a) Row-by-row scanning; (b) Column-by-column scanning; (c) 4th order neighborhood

These error measures, i.e., the phase detector outputs of the PLL, from 8 neighboring samples are averaged, and then, applied to the loop filter in order to update the phase estimate of the current center pixel. In the mean time, the neighboring pixels are also updated with respect to the center pixel. As the second step, the center pixel itself is used as the next reference input of the PLL. That is, each pixel is updated according to its 8 neighboring pixels and each neighboring pixel is updated according to different center pixels. For each pixel, the phase updates are temporarily stored in memory and then they are averaged for the final update. This operation is repeated $\mathrm{n}$ times until the phase is unwrapped.

This approach is advantageous due to the independence of unwrapping operation from the scanning direction. This eliminates the propagation of unwrapping error caused by a singularity point along the integration path.

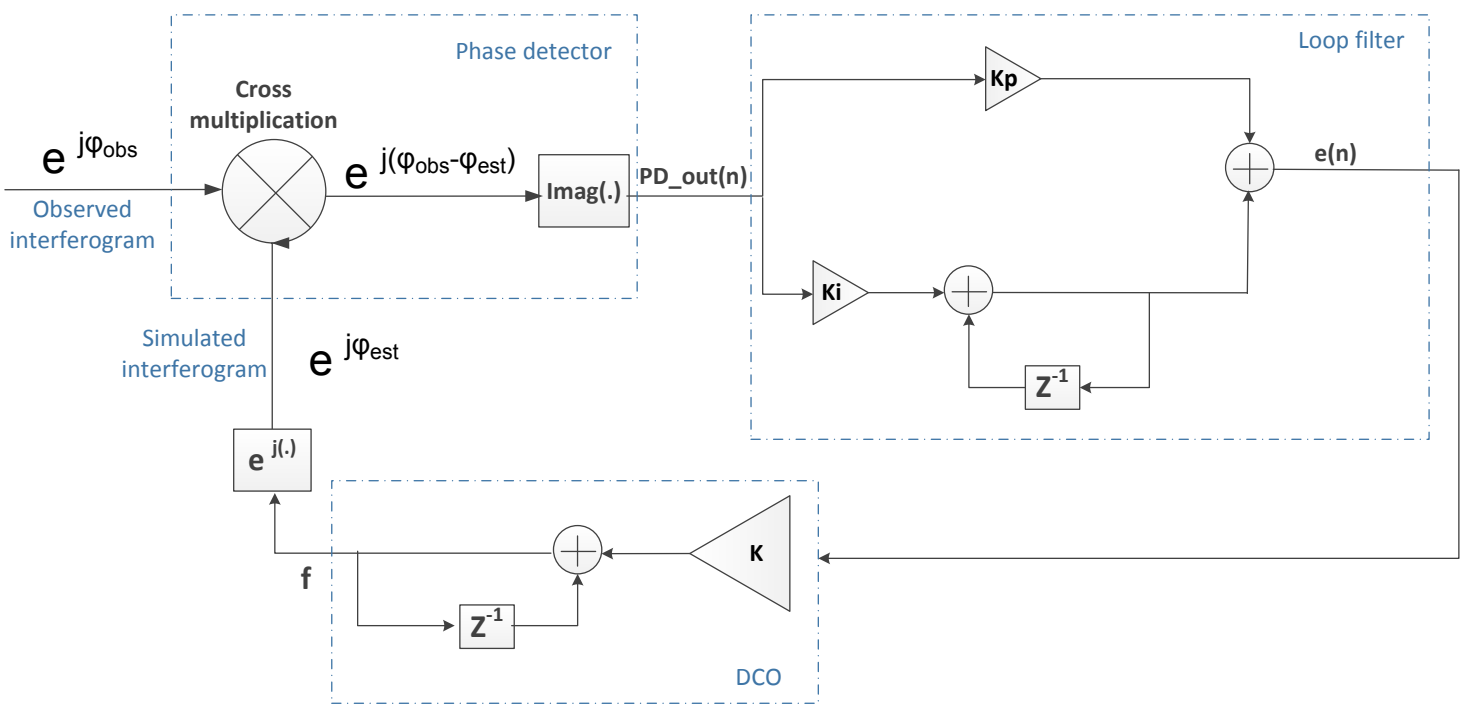

Figure 3. Second order PLL

\section{EXPERIMENTAL RESULTS}

The performance of the proposed approach is evaluated for i) a synthetic fractal Brownian surface (fBs), which is considered to be a proper model for $2 \mathrm{D}$ absolute phase, ${ }^{4}$ and ii) a real TerraSAR-X / TanDEM-X interferometric pair.

As the first synthetic data, a fBs of $257 \times 257$ with a Hurst parameter of $H=0.9$ is generated (See Fig. 4). Also, the generated fBs is corrupted by different levels of noise in order to observe the noise suppression capability 
of PLL based phase unwrapping. As it can be seen from Fig. 4 and Fig. 5, phase unwrapping is successful for a single wrapped synthetic fBs, and the noise is significantly reduced. Also, another synthetic fBs which has more wraps is generated as in Fig. 6. The results show that the method fails to unwrap successive phase wraps, while noise suppression capability is preserved.

For the real data, the contribution of the flat earth phase is removed by interferogram flattening before phase unwrapping, so the phase variations are reduced as in Fig. 7(a) and Fig. 7(b). However, the proposed method failed to completely recover the phase (See Fig. 7(c)).

The results show that the proposed method performs quite well under proper sampling condition, i.e., no aliasing, and low fringe frequency and recovers the phase gradient successfully. In case of abrupt phase changes due to the topography of terrain, the success of unwrapping operation depends on the tracking speed of PLL. In other words, if the loop bandwidth of PLL is wide enough to track these abrupt phase changes, phase unwrapping will be successful. Here, it is also important to consider the stability of the PLL and treat the trade-off between the noise suppression capability and tracking speed of PLL accordingly.

On the other hand, for surfaces with high slopes, i.e., in case of successive phase wraps, although the proposed MRF-based PLL still continues to suppress noise significantly, it fails to unwrap all the phase wraps. That is, PLL cannot add multiples of $2 \pi$ successively since it can be said to be locked to a local optimum instead of a global one. In order to overcome this bottleneck, a Metropolis-like sampling algorithm which is also used in Ref. 4 may be implemented in conjunction with the oscillator of PLL.

\section{CONCLUSIONS}

In this work, a non-causal MRF approach is adopted to PLL-based phase unwrapping. The results show that, for surfaces which do not possess large phase variations, the MRF-based PLL not only unwraps the phase but also achieves a significant noise filtering. For the case of high phase variations, this method fails to recover successive phase wraps but still continues to suppress noise. As a future work, PLL with an oscillator combined with a

Metropolis-like sampling algorithm may be used in order to overcome this problem. Nevertheless, the noise suppression capability of the proposed method may still be exploited in interferogram filtering.

\section{ACKNOWLEDGMENTS}

Thanks to German Academic Exchange Service (DAAD) for the financial support provided to first author to carry out her doctoral work at DLR, Oberpfaffenhofen.

\section{REFERENCES}

1. M. Servin and R. Rodriguez-Vera, "Two-dimensional phase locked loop demodulation of interferograms," Journal of Modern Optics, 40, no. 11, pp. 2087--2094, 1993.

2. M. A. Gdeisat, D. R. Burton, and M. J. Lalor, "Real-time fringe pattern demodulation with a second-order digital phase-locked loop," Applied Optics, 39, no. 29, pp. 5326--5336, 2000.

3. S. Geman and D. Geman, "Stochastic relaxation, Gibbs distributions, and the Bayesian restoration of images," IEEE Transactions on Pattern Analysis and Machine Intelligence, 6, no. 6, pp. 721--741, 1984.

4. G. Nico, G. Palubinskas, and M. Datcu, "Bayesian approaches to phase unwrapping: Theoretical study," IEEE Transactions on Signal Processing, 48, no. 9, pp. 2545--2556, 2000. 


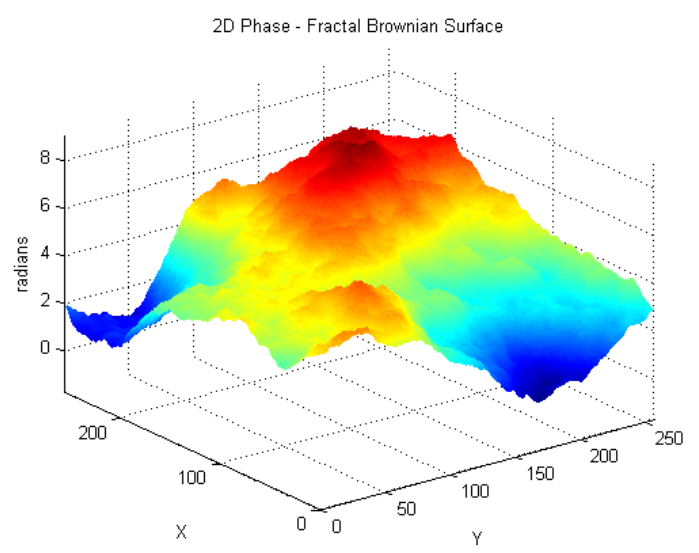

(a)

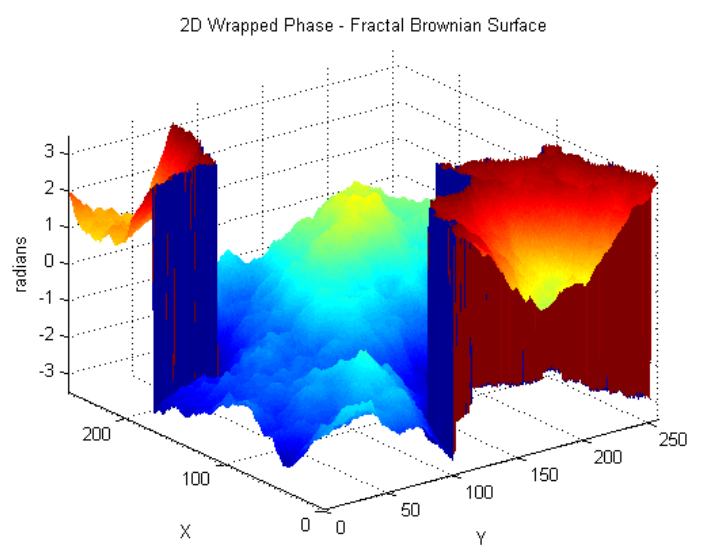

(c)

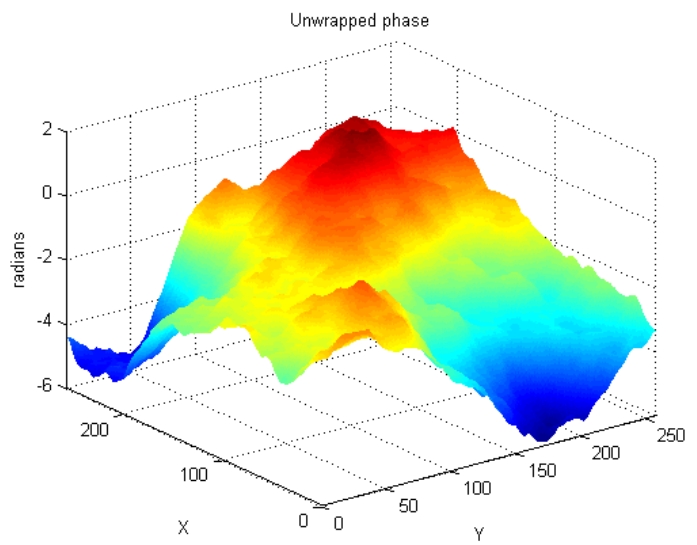

(e)

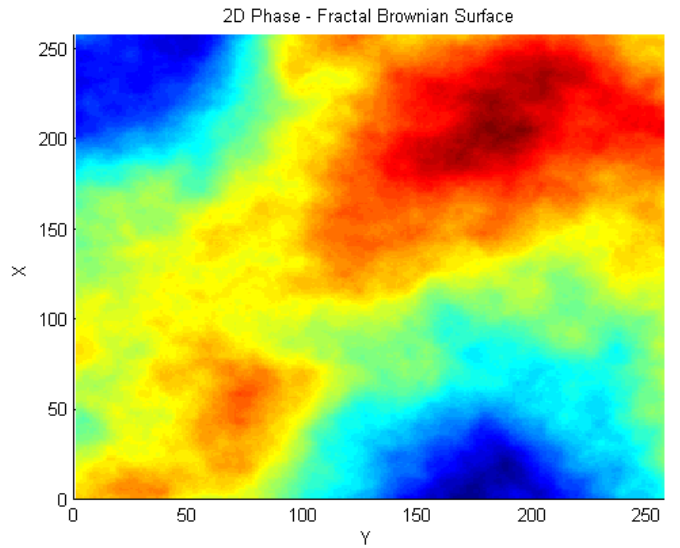

(b)

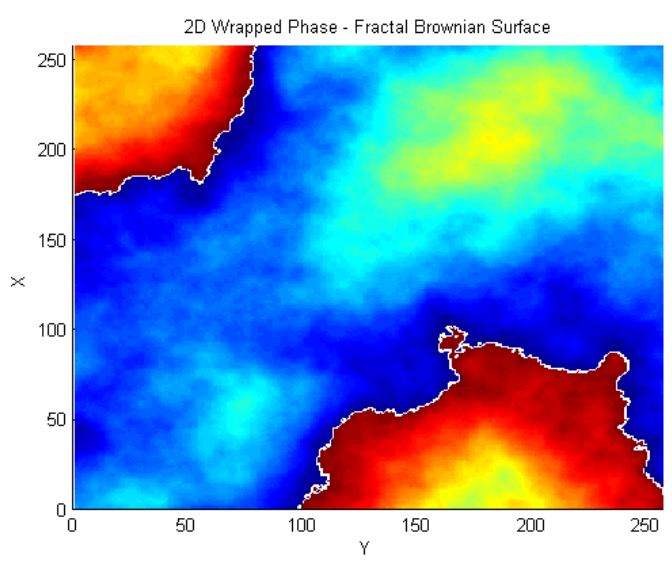

(d)

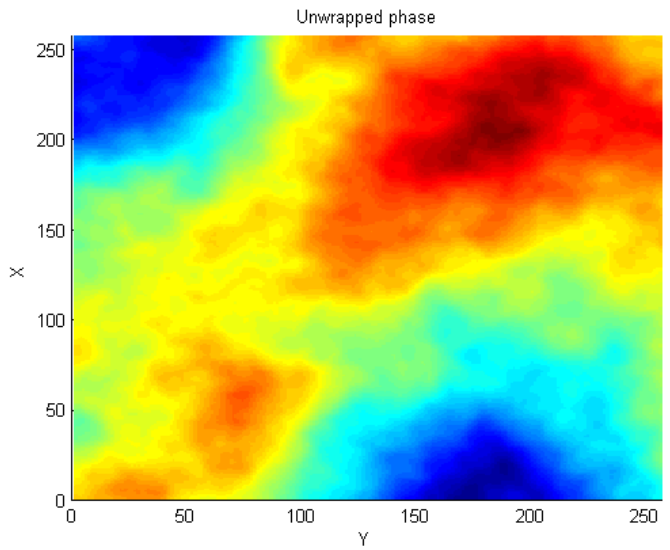

(f)

Figure 4. (a) Original fractal Brownian surface; (b) Original fractal Brownian surface (top view); (c) Wrapped phase; (d) Wrapped phase (top view); (e) Unwrapped phase using MRF-based PLL; (f) Unwrapped phase using MRF-based PLL (top view) 


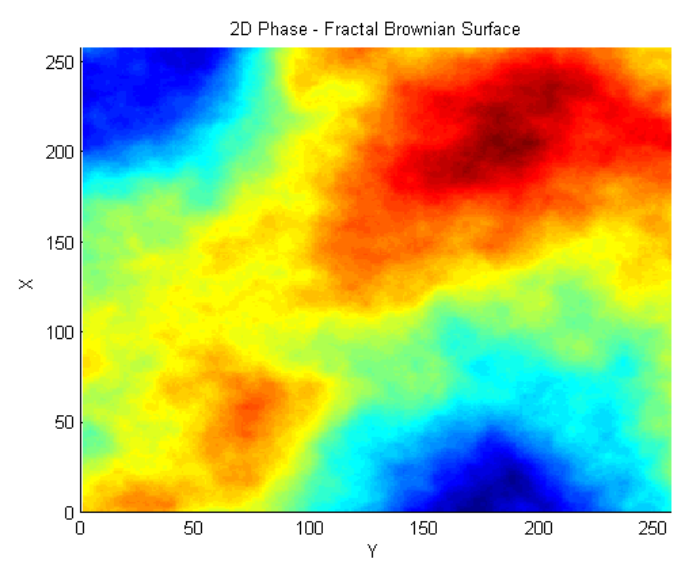

(a)

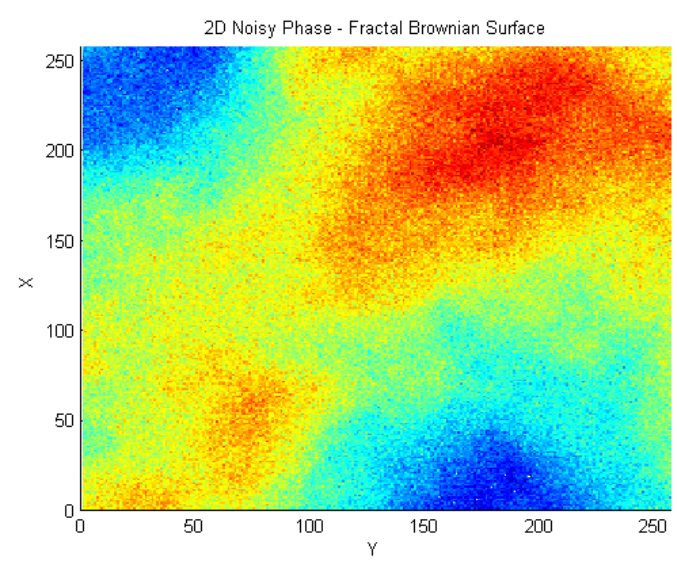

(c)

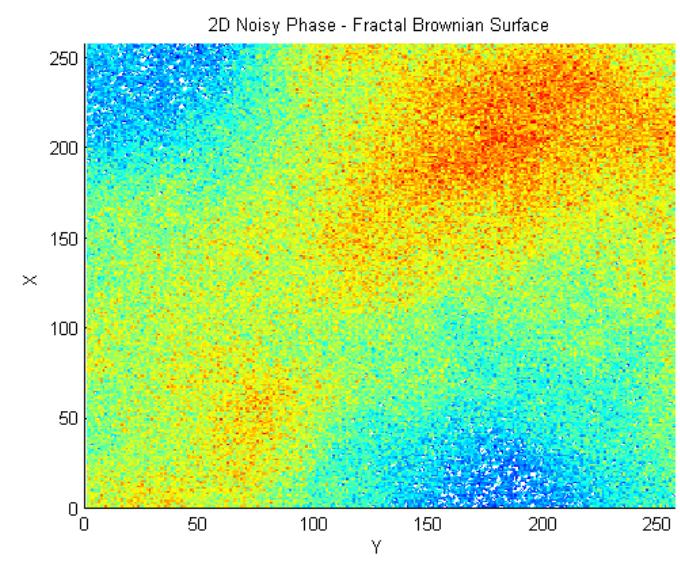

(e)

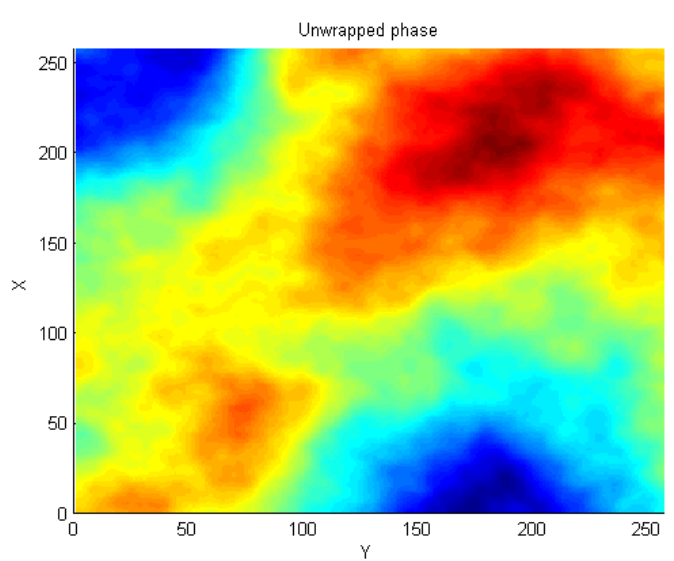

(b)

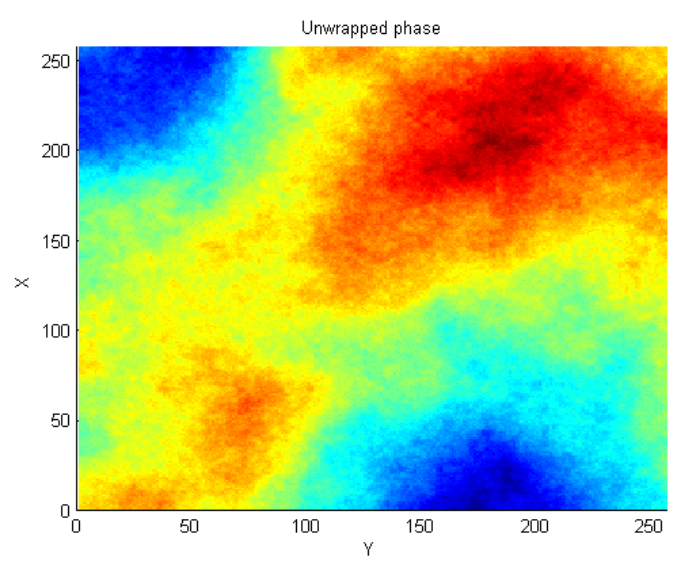

(d)

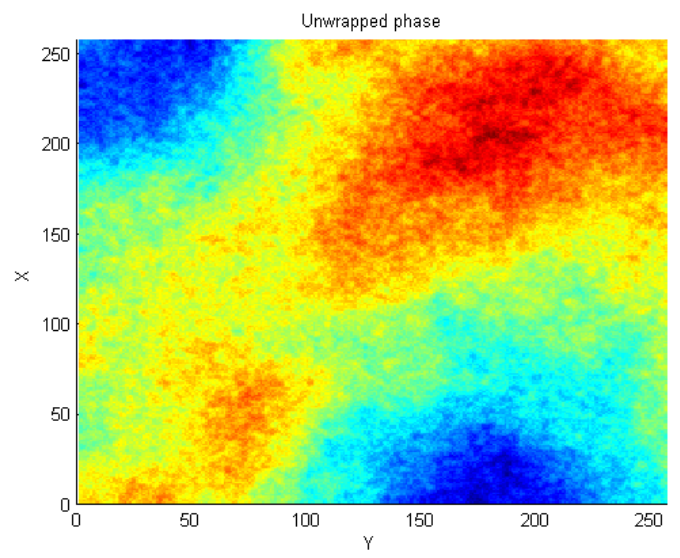

(f)

Figure 5. (a) Original fBs (no noise); (b) Unwrapped phase (no noise); (c) Original fBs with noise $\sigma=0.4$; (d) Unwrapped phase with noise $\sigma=0.4$; (e) Original fBs with noise $\sigma=0.9$; (f) Unwrapped phase with noise $\sigma=0.9$ 


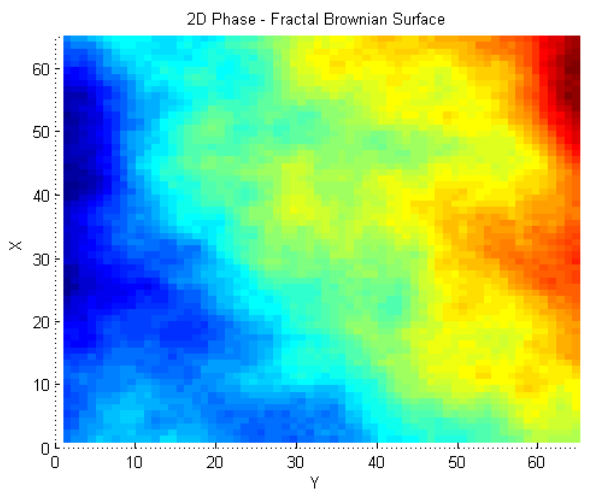

(a)

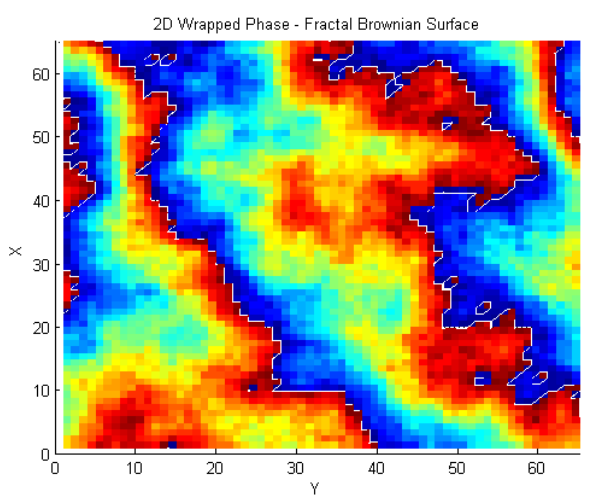

(c)

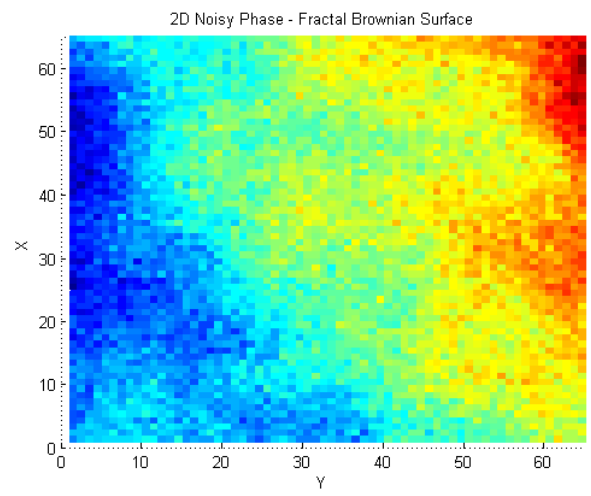

(b)

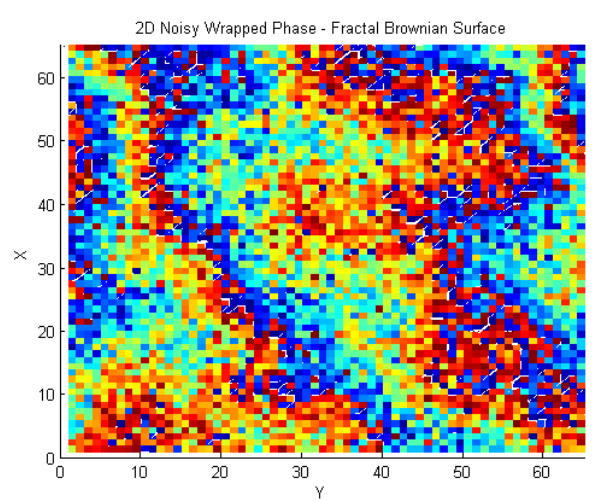

(d)

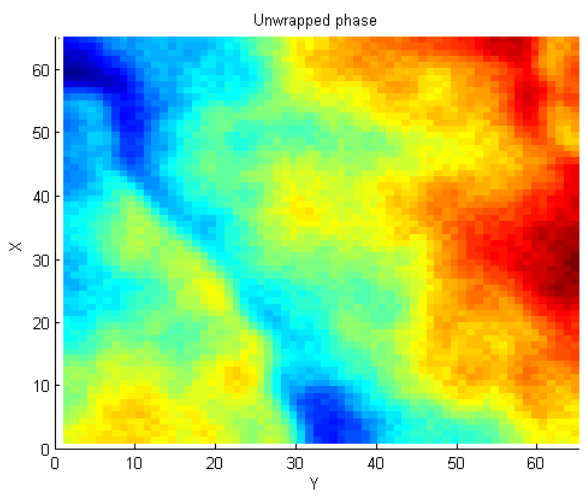

(e)

Figure 6. (a) Original fBs (top view); (b) Original fBs with noise (top view); (c) Wrapped fBs (top view); (d) Wrapped fBs with noise (top view); (e) Unwrapped phase (top view) 


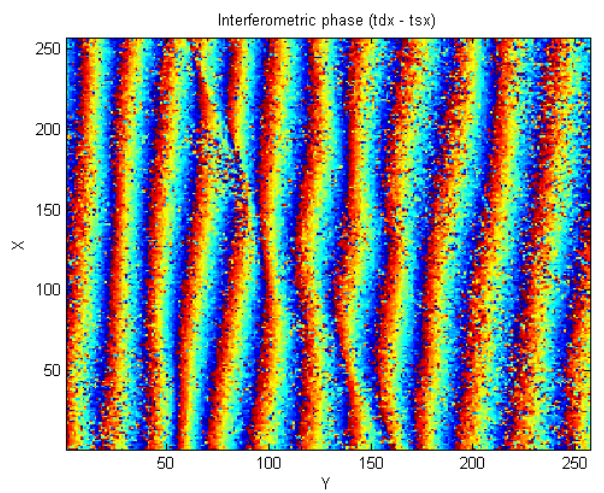

(a)

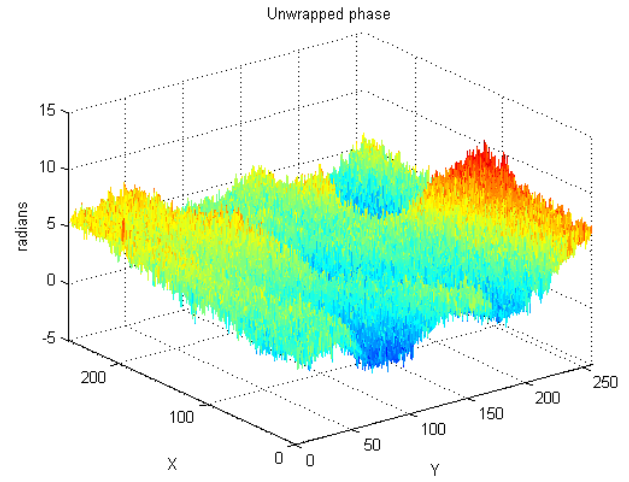

(c)

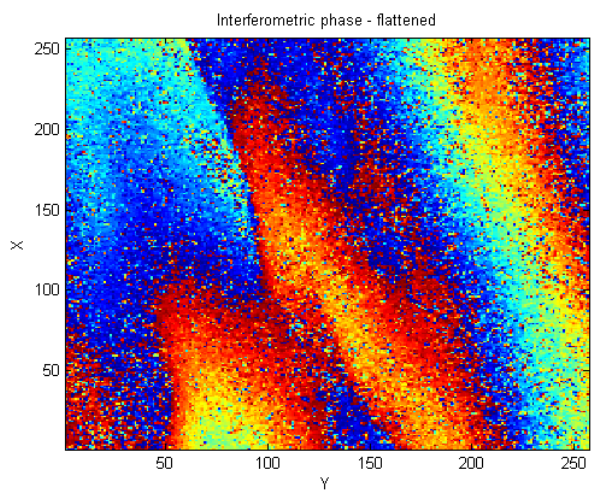

(b)

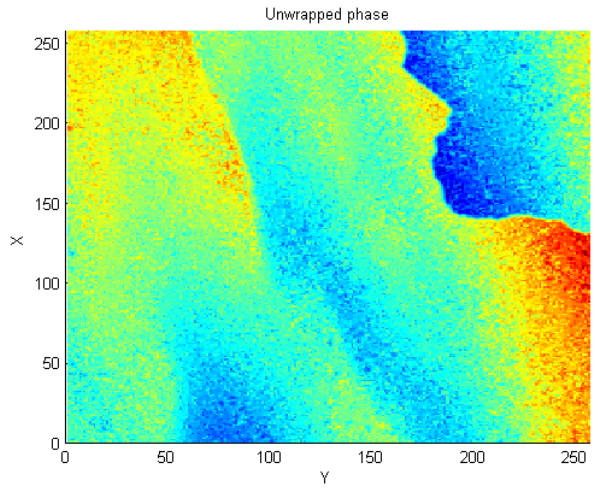

(d)

Figure 7. (a) Real interferometric TSX - TDX pair; (b) Flattened interferogram; (c) Unwrapped phase; (d) Unwrapped phase (top view) 